\title{
Unsteady flow phenomena in turbine shroud cavities
}

\section{Original article}

Article history:

Submission date: 25 November 2020

Acceptance date: 12 August 2021

Publication date: 19 October 2021

This is the updated version of a paper originally presented at the Global Power and Propulsion Virtual Technical

Conference, GPPS Chania20, Sept. 7-9, 2020.

\section{D) Check for updates}

*Correspondence:

TK: kluge@tfd.uni-hannover.de

\section{Peer review:}

Single blind

\section{Copyright:}

(c) 2021 Kluge et al. @ This is an open access article distributed under the Creative Commons Attribution License (CC-BY 4.0), which permits unrestricted use, distribution, and reproduction in any medium, provided the original work is properly cited and its authors credited.

\section{Keywords:}

unsteady flow; cavities; labyrinth seals; turbines; flow instabilities

\section{Citation:}

Kluge T., Lettmann I. S., Oettinger M., Wein L., and Seume J. R. (2021). Unsteady flow phenomena in turbine shroud cavities. Journal of the Global Power and Propulsion Society. 5: 177-190.

https://doi.org/10.33737/jgpps/141211

\author{
Tim Kluge ${ }^{1, *}$, Iris S. Lettmann ${ }^{1}$, Marcel Oettinger ${ }^{1}$, Lars Wein ${ }^{1}$, Joerg R. Seume ${ }^{1}$ \\ ${ }^{1}$ Institute of Turbomachinery and Fluid Dynamics, Leibniz University Hannover, An der Universität 1, \\ DE-30823 Garbsen, Germany
}

\begin{abstract}
This paper presents those flow parameters at which coherent structures appear in the blade tip cavities of shrouded turbine blades. To the authors' knowledge, this is reported for the first time in the open literature. The unsteady flow in a shroud cavity is analysed based on experimental data recorded in a labyrinth seal test rig. The unsteady static wall pressure in the shroud cavity inlet and outlet is measured using time-resolving pressure sensors. Sensors are located at staggered circumferential positions to allow cross-correlation between signals. The unsteady pressure signals are reduced using Fourier analysis and cross-correlation in combination with digital filters. Based on the data, a theory is formulated explaining the phenomena reflected in the measurements. The results suggest that pressure fluctuations with distinct numbers of nodes are rotating in the shroud cavity outlet. Moreover, modes with different node numbers appear to be superimposed, rotating at a common speed in circumferential direction. The pressure fluctuations are not found at all operating points. Further analysis indicates that the pressure fluctuations are present at operating points matching distinct parameters correlating with the cavity flow coefficient. Unsteady RANS simulations predict similar flow structures for the design operating point of the test rig.
\end{abstract}

\section{Introduction}

The design of modern, efficient turbomachinery requires the consideration of secondary flow during early design stages (Cumpsty, 2010). In order to do so, the numerical tools used during the design are subject to continuous improvements. The design tools must account for unsteady flow phenomena in addition to steady-state or time-averaged secondary flow.

This paper presents and analyses unsteady flow phenomena measured in the cavity outlet of a turbine shroud labyrinth seal. Unsteady pressure fluctuations such as those reported in this paper have often been found in rim seal cavities. However, there are currently no studies the authors could find in open literature focussed on similar phenomena inside shroud cavities at turbine blade tips. The occurrence of the unsteady fluctuations, as evident in the pressure spectrum measured in the data at hand, may be explained satisfactorily by means of a simple kinematic theory presented in this paper.

In analysing the data, the following questions are addressed: (1) What is the cause of the unsteady pressure fluctuation in the labyrinth seal cavity outlet? (2) Which parameters influence the occurrence of the unsteady phenomenon? (3) Can the phenomenon be reproduced in CFD, i.e. modelled correctly? 
There is previous research, both experimental and numerical, into unsteady flow phenomena in rim seal cavities in axial turbines. However, the similarity of unsteady flow phenomena observed in the rim cavities to those in tip shroud cavities reported in this paper is remarkable as shown in the results section below.

Beard et al. (2017) conducted an experiment on a rim seal test rig operated without mainstream air and without blades in the main channel. They identify a flow structure with 27 to 29 lobes in the rim seal rotating at an angular speed of $\omega_{s} / \Omega \approx 0.8$, i.e. approximately $80 \%$ of the rotor speed. The study shows that increasing the rotational speed or the sealing mass flow reduces the number of lobes. The corresponding numerical studies were conducted by Gao et al. (2017). LES and URANS simulations in the latter study show reasonably good agreement with steady-state measurements within the disc cavity when compared with experimental data. Unsteadiness at a similar distinct peak frequency can only be found in the LES. The authors suspect the discrepancies in number of lobes, structure, and rotational velocity to be caused by the limited circumferential sector of the numerical model or other approximations.

Boudet et al. (2006) investigate the rim seal of an axial turbine by means of numerical simulations. The results show fluctuations at frequencies lower than the blade passing frequency. Further simulations show that the instability develops inside the seal cavity. The authors associate the phenomenon with Taylor-Couette instability, as the conditions for the flow regime are met inside the rim seal. There is no information concerning the rotational frequency of the fluctuations.

Numerical simulations with two set-ups are conducted by Rabs et al. (2009). In the first numerical simulation, the guide vanes and blades are omitted, resulting in a simplified turbine rim cavity model. The pressure distributions show regions with high and low pressure in circumferential direction due to Kelvin-Helmholtz vortices. In a second simulation, a full model of a 11/2-stage turbine is modelled. Here, the vortices appear at high non-dimensional sealing air mass flow rates and are suppressed by the interaction of the guide vanes and the blades for low and medium flow rates. The magnitude and frequency of the occurrence is decreased compared to the simplified model.

Boutet-Blais et al. (2011) conducted numerical simulations of a simplified turbine rim seal environment without blade and vanes. Large-scale structures with 29 regions of high and low pressure inside the seal cavity were identified, which rotate at $\omega_{s} / \Omega \approx 0.79$. The study notes that the frequency corresponding to this effect is far more energetic than that of the blade passage. Furthermore, it shows that the presence of these large-scale structures directly influences the seal effectiveness.

Cao et al. (2004) study the interaction of rim seal and annulus flows by means of numerical computations as well as experiments. They identify unsteady flow structures with 11-14 nodes inside the rim seal rotating at slightly less than rotor speed. The unsteadiness causes peaks in the spectrum in the range of $600-800 \mathrm{~Hz}$. Further examination suggests that the number of nodes around the annulus varies over time.

Horwood et al. (2018) conducted a combined experimental and computational investigation. The study also identified structures, which rotate at an angular velocity of $\omega_{s} / \Omega=0.81 \ldots 0.94$ and $\omega_{s} / \Omega=0.92 \ldots 1.03$ in simulation and experiments, respectively. The study names Kelvin-Helmholtz instabilities as the cause. In a later publication, Horwood et al. (2020) examine further operating conditions. The peak-frequency and amplitude of the phenomena are found to be dependent on the sealing purge flow. The statement concerning the cause of the large-scale structure is amended to include Taylor-Couette instabilities.

Hualca et al. (2020) state that the positions of vanes and the presence of blades affect the rotating structures. The frequency of signal peaks is weakly dependent on the vane position and the sealing mass flow. Without the rotor blades, structures with 34 to 40 lobes that rotate with $\omega_{S} / \Omega=0.71 \ldots 0.98$ were identified. With rotor blades, the number of lobes is reduced to 22 to 28 and the structures rotate with $\omega_{S} / \Omega=0.96 \ldots 1.12$.

Numerical simulations by Jakoby et al. (2004) show three low pressure regions inside the cavity, which rotate with $\omega_{S} / \Omega=0.80$. With an increase in the cooling air mass flow, the phenomenon disappears. Similar findings are presented by Julien et al. (2010). Large-scale structures with 30 lobes are detected for no and low purge flow conditions. The coherent structure in this study rotates at $\omega_{S} / \Omega=0.87$ and 0.80 , respectively. In the case of a high purge flow condition, only smaller pressure pulses deeper inside the cavity instead of large-scale structures can be found.

Schaedler et al. (2016) also detect low frequency pressure fluctuations in an experimental and numerical investigation. The structures are dependent on the purge mass flow as the band of frequencies with elevated amplitudes is shifted to higher frequencies when the purge mass flow is increased. It is not present for the highest injection rate. The number of lobes ( 8 and 22 , respectively) and angular velocity $\left(\omega_{S} / \Omega=0.82\right.$ and 0.93 , respectively) are derived from the numerical simulation. The pressure fluctuations have a significant impact on the noise level as an increase of up to $18 \mathrm{~dB}$ can be detected when the structures are present.

Using URANS-based CFD-simulations of a stepped labyrinth seal, Wein et al. (2018) found a nodal structure rotating at approximately $76 \%$ of the shroud rotational speed. The rotating nodes were found to cause a variation in size of the cavity vortices in the swirl chamber between two sealing fins. The nodal structures were found to 
be suppressed at some operating points. While this was attributed to a large dimensionless cooling flow rate $C_{W}>18,000$ in one case, the cause was not entirely clear in a second case.

It may be noted that some of the studies mentioned above show that rotating structures affect the sealing efficiency and lead to ingestion of hot air into the rim seal cavity. This serves to emphasise the importance of understanding the unsteady secondary flow in turbomachinery.

The following paper is organised into four main sections: In the first section following the introduction the test facility, instrumentation, and the numerical model are described. The theoretical background is outlined in the second section, detailing a simple kinematic model to explain the data presented in the results. Before the results are presented, the analytical methods used in this study are defined in the third section of this paper. In the fourth section, the experimental results are presented and discussed. The data is also compared to CFD simulations to verify to which extent the observations can be reproduced in numerical models.

\section{Experimental facilty \& numerical model}

The data presented in this paper was recorded using a labyrinth seal test rig with a rotating seal carrier disk. The flow tested is limited to shroud leakage flow, isolating the cavity flow from interaction with the main flow in a turbine. The test rig is introduced in more detail in Kluge et al. (2019). It is designed to mirror the shroud cavity geometry of the axial turbine in operation at the Institute of Fluid Dynamics and Turbomachinery at Leibniz University Hannover. The characteristic turbine parameters on which the test rig is based may be found in Biester et al. (2012) and Henke et al. (2012, 2016).

\section{Design}

A longitudinal section of the rig is presented in Figure 1. The flow path is shaded in blue colour and the flow direction is indicated by arrows. Air enters the test rig radially through a volute, which imposes a constant swirl

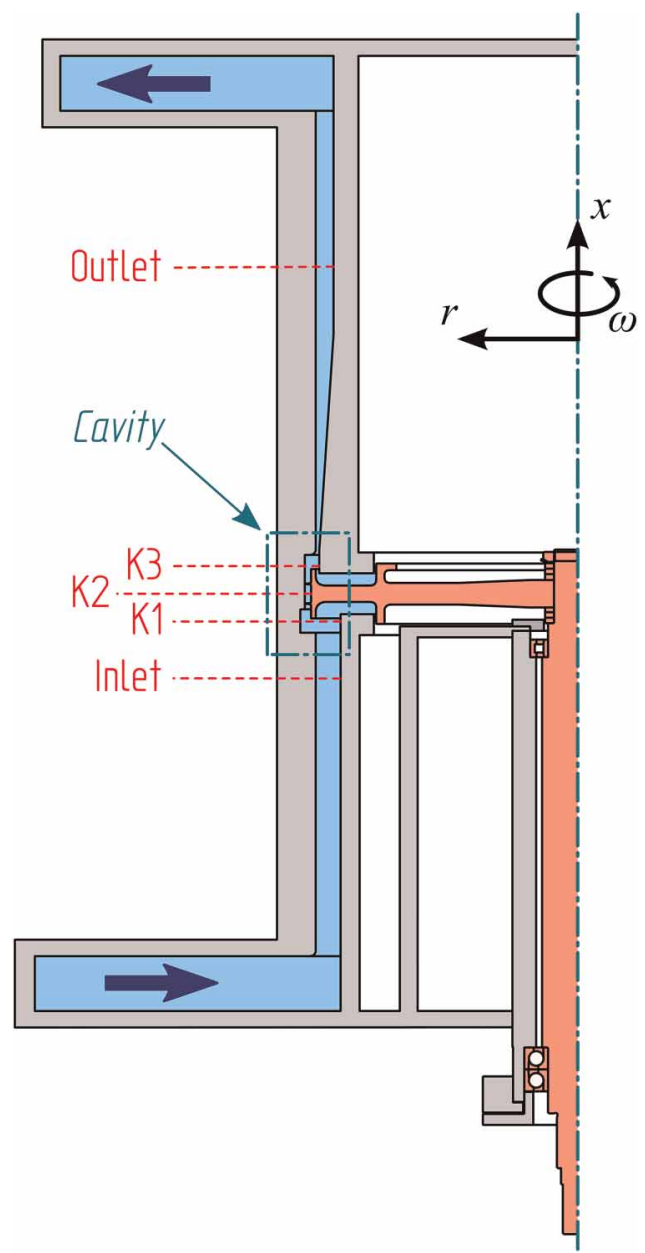

Figure 1. Longitudinal section of the labyrinth test rig (enlarged detail of shroud cavity in Figure 2). 
angle flow. The outlet is formed by a second volute, which minimises the circumferential inhomogeneity (Kluge et al., 2019). The flow enters the vertical annular flow channel and passes the labyrinth seal formed by an interchangeable outer contour ring and a disc carrying the labyrinth seal. The rotating components of the test rig are shaded red. A rotating disc, driven by an electric motor, serves as labyrinth seal carrier without blades. The preswirl of the flow and the rotational speed of the disc in combination result in flow kinematics similar to those in the turbine test rig (Henke et al., 2016). In both rigs, the cavity geometry is identical, the pressure ratio across the labyrinth seal is the same and, thus, the leakage mass flow may be expected to be equal, and the rotational speeds are the same. The swirl angle of the flow upstream of the cavity inlet is the same within $\pm 2.5^{\circ}$.

The inlet and outlet planes represent the beginning and end of the control volume in all CFD simulations. The boundary conditions are measured in these locations. The labyrinth seal cavity is subdivided into the cavity inlet (K1), swirl chamber (K2), and cavity outlet (K3), cf. Figure 1.

The entire test rig between inlet and outlet volute is rotationally symmetric. The flow path is free from obstructions such as struts to eliminate external excitation of unsteadiness.

\section{Instrumentation}

Detailed measurements of pressure and temperature are recorded in the cavity inlet (K1) and outlet (K3) as well as the swirl chamber (K2), cf. Figure 2. The instrumentation is listed in more detail in Kluge et al. (2019).

Total and static pressure as well as total temperature are measured in the inlet and outlet plane of the test rig. All data is recorded synchronously to provide the boundary conditions for the analysis of time-resolved pressure measurements.

To record unsteady flow phenomena inside the inlet and outlet cavities, both are instrumented with timeresolving pressure sensors (Kulite XCQ-062) in the axial positions K1.1 and K3.3, cf. Figure 2. Two circumferential locations are available in the cavity inlet spaced circumferentially $180^{\circ}$ apart. Preliminary measurements have shown that no significant source of unsteadiness is present inside the cavity inlet. The cavity outlet is instrumented at $\alpha=0^{\circ}, 7.5^{\circ}, 22.5^{\circ}$, and $57.5^{\circ}$ circumferentially. An additional position at $180^{\circ}$ is available. The operating point in the experiment is defined by the reduced mass-flow rate and the reduced rotational speed to obtain constant axial and rotational Mach numbers independent from ambient conditions.

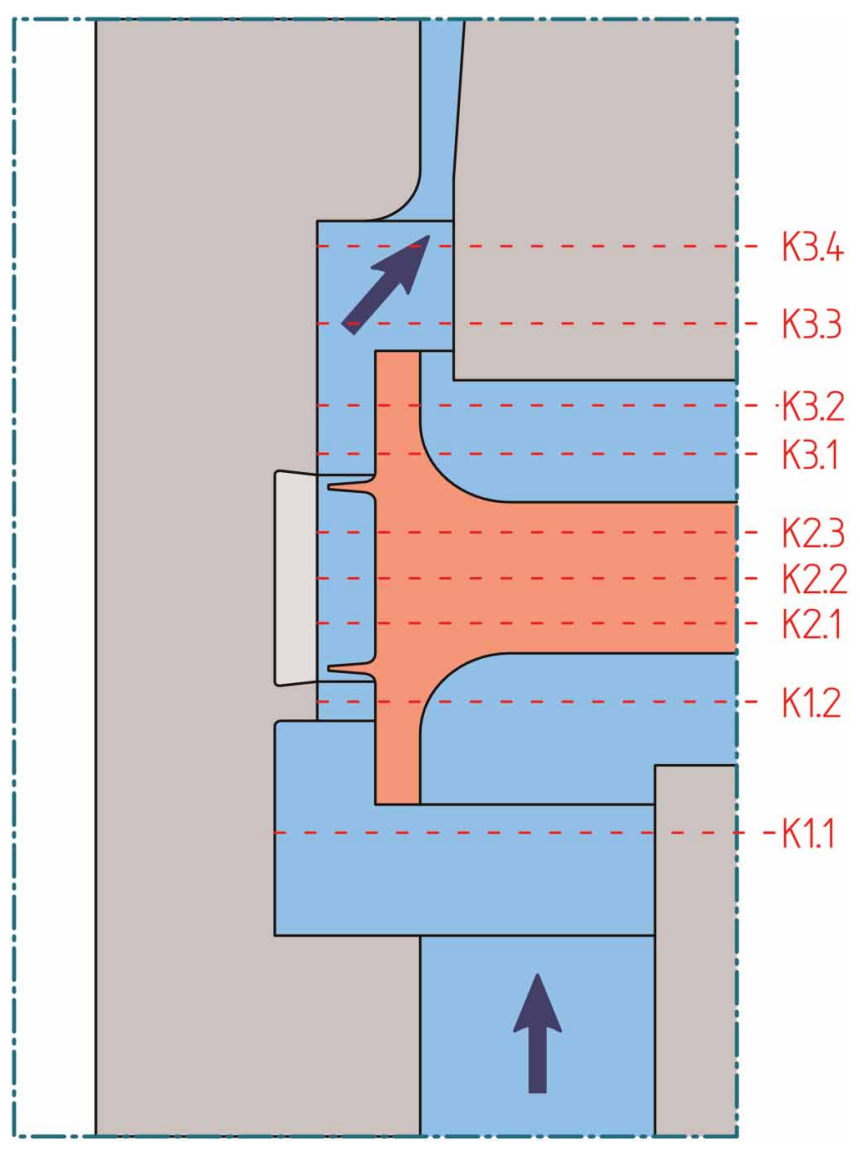

Figure 2. Longitudinal section of the labyrinth seal cavity (cf. Cavity in Figure 1). 


\section{Numerical set-up}

Time-resolved simulations in this study are implemented using TRACE (Kügeler, 2004; Nürnberger, 2004), a flow solver for turbomachinery applications developed by the Institute of Propulsion Technology of the German Aerospace Center (DLR) in cooperation with MTU Aero Engines AG. At the inlet, circumferentially averaged radial profiles of the total pressure, total temperature, and flow direction derived from the experiment are specified. At the outlet, the circumferentially averaged static pressure with a radial equilibrium boundary condition is defined. The boundary layers are assumed fully turbulent, i.e. transitional effects are neglected and the walls are assumed to be adiabatic. To minimise discretisation errors, a second order accurate Fromm scheme (Darwish, 1993) is selected for the discretisation of spatial derivatives of the convective term. The Fromm scheme is bounded by the van Albada limiter (van Albada et al., 1982) and for the viscous fluxes a second-order accurate central difference scheme is used. The temporal derivatives are discretised by a second order accurate backward Euler time scheme.

To account for turbulence, the $k-\omega$ turbulence model described by Wilcox (1988) in combination with the stagnation point correction proposed by Kato and Launder (1993) is applied. This model produces results, which are in good agreement with measurement data of losses and pressure drop across the labyrinth seal in steady-state simulations (Wein et al., 2020).

The domain and spatial discretisation is defined in Kluge et al. (2019) in more detail. Periodic boundary conditions can lead to erroneous predictions of unsteady flow phenomena in labyrinth seals (Wein et al., 2018). Consequently, the full annulus of the rig is modelled in this study. In the direction normal to the wall $y^{+} \leq 1$ is adhered to and the cell size increases with a factor of 1.2. Due to the high acceleration, turning, and detachment of the flow, appropriate resolution and grid quality in the labyrinth clearance is necessary. Therefore, the nondimensional axial cell size in the clearance is $(\Delta x / c) \leq 0.06$. In the rest of the domain, an axial cell size of $(\Delta x / c) \leq 0.6$ is selected and three cells per $1^{\circ}$ are used in circumferential direction. A grid convergence study was performed in accordance with Kluge et al. (2019).

\section{Theory}

The following kinematic model serves to explain the spectral footprint of the unsteady measurements reported in the results section of this paper. The spectrum found in the data shows periodic signal components, which are non-harmonic to the rotor frequency (as shown in an exemplary spectrum below). The model described here is referred to during the analysis of the data.

The model consists of rotating nodal structures. Multiple modes are superimposed while rotating at a common speed. The physical nature of the nodes themselves is arbitrary, however, in the present data they manifested as pressure fluctuations. An example of a single mode is shown in Figure 3. This structure consists of a distinct number $N$ of pressure lobes equally spaced over the circumference (spacing $\beta$ ). It rotates at an angular speed $\omega_{s}$.

Inside the cavity, a minimum of two sensors are mounted, spaced at a given angle $\alpha$. The frequency

$$
f=\frac{1}{\Delta t_{\beta}}
$$

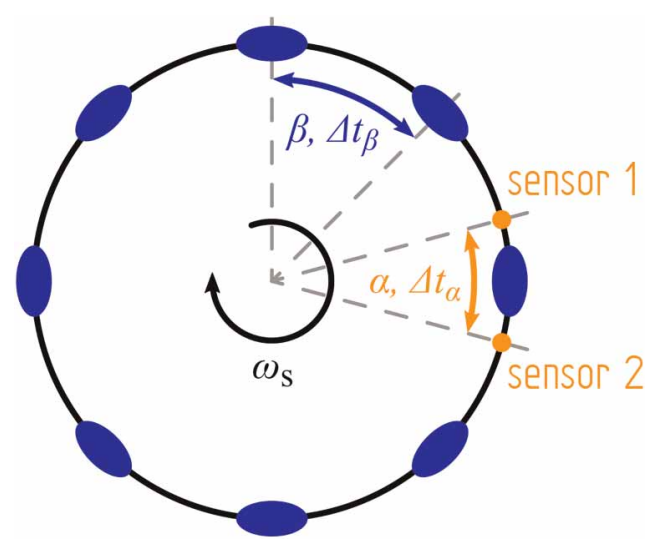

Figure 3. Schematic of the rotating coherent structure with $N=8$ nodes, angular relations, and exemplary sensor positions. 


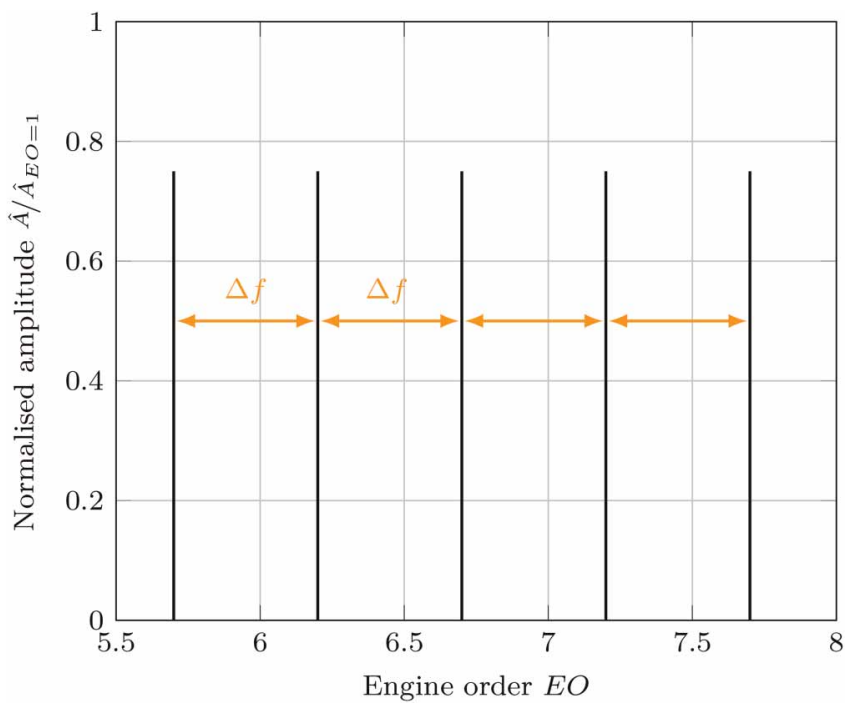

Figure 4. Spectral response due to five superimposed nodal structures with $N_{i}=N_{1}+k$ rotating at a common speed $\omega_{s}$.

may be detected in unsteady pressure traces recorded by both sensors. The time lag while one lobe passes through the angle $\beta=(2 \pi / N)$ is defined by

$$
\Delta t_{\beta}=\frac{\beta}{\omega_{s}}
$$

Accordingly, the time lag

$$
\Delta t_{\alpha}=\frac{\alpha}{\omega_{s}}
$$

defines the duration of the passage of one lobe through the angle $\alpha$. The latter defines also the time shift between the two signals. The time lags $\Delta t_{\alpha}$ and $\Delta t_{\beta}$ are determined by cross-correlating the two signals.

Rotating periodic structures cause a distinct frequency response. When multiple modes, which differ only in their number of pressure lobes $N_{i}$ but not in $\omega_{s}$, are superimposed, the sensors detect a corresponding number of distinct frequencies. In this case, the amplitude spectrum of the signal trace shows peaks, which are evenly spaced by

$$
\Delta f=f_{2}-f_{1}=\frac{\omega_{s}}{2 \pi}
$$

where the individual frequencies are given by

$$
\begin{gathered}
f_{i}=\frac{\omega_{s}}{2 \pi} \cdot N_{i} \\
N_{i}=N_{1}+k \quad \text { with } k \in \mathbb{Z}
\end{gathered}
$$

An example of such a spectrum is given in Figure 4.

\section{Methodology}

The methods used for the analysis of data in this study are defined in this section. They are applied to experimental as well as numerical data. First, all data is low-pass filtered for anti-aliasing by a first-order Butterworth filter with a cut-off frequency of $90 \mathrm{kHz}$, i.e. less than half of the sampling frequency of $200 \mathrm{kHz}$. Second, spectral analysis of the data is performed by means of a fast Fourier transform with a von-Hann window function. 
The number of nodes and rotational speed of individual rotating modes are identified by cross-correlating signals from different sensors. An example of the cross-correlation is given in Figure 5. To isolate individual modes, the signals are filtered by tenth-order Butterworth band-passes.

By cross-correlation, $\Delta t_{\alpha}$ is determined as the first maximum to the right of the origin. $\Delta t_{\beta}$ is equivalent to the time lag between two adjacent maxima (cf. Figure 5). In the case of large numbers of nodes or large angles between correlated sensors, i.e. $\alpha>\beta$, erroneous readings occur. In this case, the true time lag of the two signals is given by

$$
\Delta t_{\alpha}=\frac{\alpha}{\omega_{s}}+k \cdot \Delta t_{\beta} \quad \text { with } k \in \mathbb{N}
$$

While $\Delta t_{\beta}$ can be identified unambiguously in the cross-correlation results, the readings of $\Delta t_{\alpha}$ may be ambiguous. The latter value must be confirmed by correlating decreasing angles between two sensors. It is most likely that the smallest spacing between two sensors gives the true value for $\Delta t_{\alpha}$.

As indicated in the previous section, multiple modes with varying nodal numbers are superimposed to produce the spectral response found in the data. To determine whether truly synchronous superposition or varying nodal numbers occur, the variation of the spectrum over time is visualised in the form of waterfall diagrams. Ideally, a spectral analysis for each rotor revolution would be conducted. However, due to the high angular velocity, a sufficient resolution of the target frequency band cannot be achieved. Instead, the spectral analysis is performed for a sliding window of the signal trace. A length of 50 revolutions leads to a sufficiently fine resolution of $\Delta f \approx 1.5 \mathrm{~Hz}$ (cf. Equation 9).

In an effort to analyse the parameters influencing the occurrence of unsteady pressure fluctuations presented in the results, the theoretical axial velocity $c_{a x}^{*}$ of the flow inside the seal clearance is determined. To this end, the following approximations are necessary. The clearance is calculated a priori, taking into consideration the centrifugal strain of the disk at each operating point (cf. Kluge et al., 2019). Due to the constraint of the leakage jet by vortices forming in the labyrinth seal, the annulus area may be approximated by the area in the seal clearance. The temperature in the shroud area (K2.2) is approximated by the mean value of the temperatures inside the cavity inlet (K1.1) and outlet (K3.3). With the pressure and mass flow measured in situ, the theoretical incompressible velocity in axial direction may be calculated. The cavity flow coefficient

$$
\varphi_{\mathrm{cav}}=\frac{c_{\mathrm{ax}}^{*}}{U}
$$

is defined, relating the theoretical incompressible axial velocity to the circumferential speed at the seal clearance. This parameter has proven to be well suited for characterising the unsteady flow field, as will be shown in further

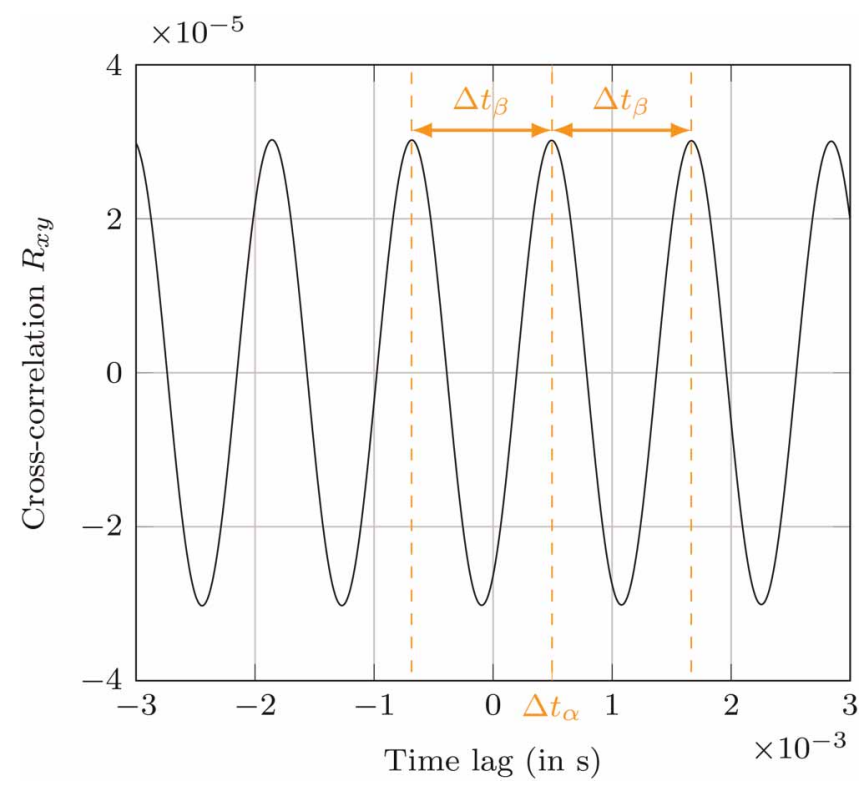

Figure 5. Cross-correlation of filtered signal traces are used to determine parameters describing unsteady phenomena. 
Table 1. Cross-correlation results at operating points featuring unsteady pressure fluctuations.

\begin{tabular}{|l|c|c|c|c|}
\hline & $\Omega / \Omega_{\mathrm{ADP}}$ & $\Pi$ & $N$ & $\omega_{\mathrm{s}} / \Omega$ \\
\hline OP 1 (ADP) & $100 \%$ & 1.280 & $16 \ldots 21$ & 0.365 \\
\hline OP 2 & $100 \%$ & 1.152 & $16 \ldots 19$ & 0.394 \\
\hline OP 3 & $50 \%$ & 1.044 & $15 \ldots 18$ & 0.397 \\
\hline
\end{tabular}

detail below. This parameter is not to be confused with the integral stage flow coefficient at the blading of a turbine.

\section{Results and discussion}

Measurement data of multiple operating points is available for analysis. The unsteady phenomenon, which is the subject of this study, is present in the spectrum at three operating points (cf. Table 1). A summary of all operating points, including those, which feature no unsteady fluctuations, is given in Table 2 in the appendix. In the first part of this section, the results of the aerodynamic design operating point (ADP) are presented in detail. The results for alternative operating points are presented more briefly further below, highlighting differences and common aspects of the rotating structures found.

\section{Design operating point}

Figure 6 shows the amplitude spectrum of one sensor in the cavity outlet at the design operating point. Various signal components are evident in distinct peaks in the spectrum. The peaks at the integer multiples of the engine order (EO) are associated with harmonic signal components caused by the revolving rotor. In the band of $E O=5.5$ to $E O=8$ there are multiple peaks that cannot be explained as easily and indicate unsteady and nonharmonic flow phenomena. A closer look at this frequency band reveals that these peaks are equidistantly spaced by $\Delta f \approx 42.6 \mathrm{~Hz}$ and form the distinctive pattern introduced above (cf. Figure 4). Each peak in the amplitude spectrum corresponds with one modal structure with a distinct number of nodes. Further analysis by crosscorrelation shows the number of nodes varies from $N=16$ to $N=21$. The significance of this frequency response is evident, as the amplitude of the peaks is $40 \ldots 80 \%$ of the amplitude at an engine order of unity. The spectral response of all three other sensor traces in the cavity outlet at K3.3 (Figure 2) is similar. Hence, the extent of this phenomenon is not spatially confined to one sensor location.

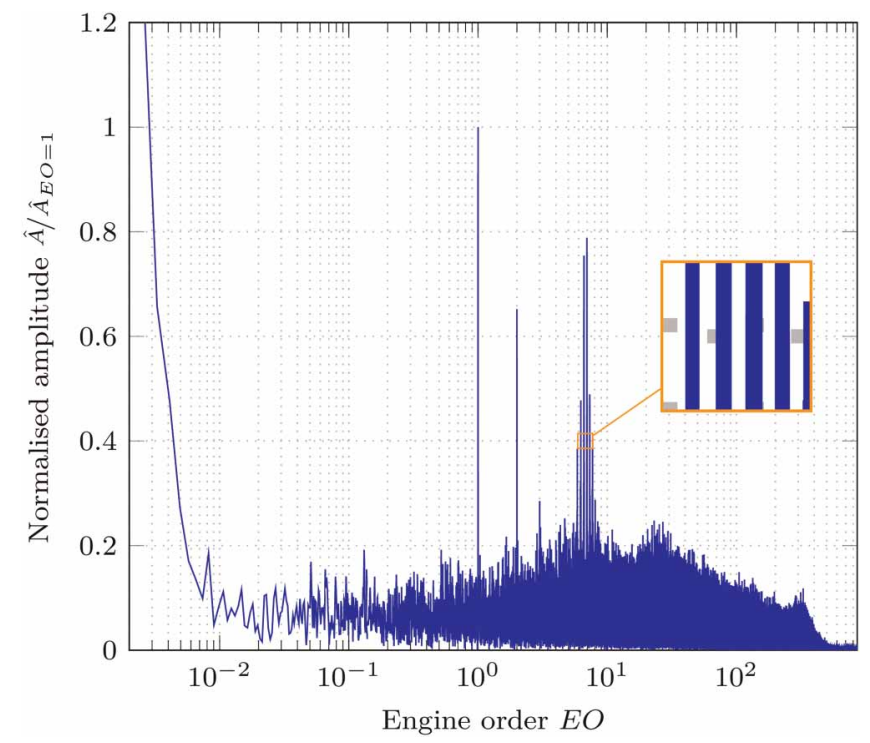

Figure 6. Amplitude spectrum of one sensor in the cavity outlet at OP 1. 
With the four sensors, six pairwise cross-correlation combinations are available to identify the rotating structures in the cavity. Figure 7 shows the results for one isolated peak in the amplitude spectrum at the ADP, i.e. for one of the superimposed nodal modes. The first five maxima to the right of the origin are plotted to account for the relation in Equation 7. Nevertheless, it appears plausible that the smallest sensor spacing yields the true result for $\Delta t_{\alpha}$. This interpretation is confirmed by the high correlation coefficient $R^{2}=1$ of the regression function added to the plot. The slope of the regression function itself is equivalent to the angular speed of the mode $\omega_{s} \approx 0.365 \Omega$. Thus, the coherent structures found in this study rotate at a significantly lower relative speed than those in rim seals reported in open literature.

The development of the rotating structures over time is analysed in a waterfall diagram in Figure 8. Five vertical lines are distinctly identifiable, which correspond to the frequency pattern in the amplitude spectrum in Figure 6, i.e. the rotating structures featuring different numbers of nodes as identified by cross-correlation. The data at operating points 1 and 2 suggests that multiple modes are superimposed. For operating point 3 , the data appears to suggest that only one mode is present for each point in time with the number of nodes varying. This would suggest a periodic breakdown and re-forming of these structures.

\section{Alternative operating points}

At operating point 3, there are distinct peaks in the amplitude spectrum in a lower frequency band, but at similar engine orders compared with OP 1 and 2. This is due to the reduced speed of the rotor. Four distinct modes are detected by cross-correlation with $N=15 \ldots 18$ nodes rotating at an angular velocity of $\omega_{s} \approx 0.397 \Omega$.

At operating point 2 , the frequency pattern is located in a similar frequency band $E O=5.5 \ldots 7.5$. The modes in the frequency band consist of $N=16 \ldots 19$ nodes rotating at an angular velocity of $\omega_{s} \approx 0.394 \Omega$.

The modal structure of the unsteady pressure fluctuation is quite similar when present, i.e. for the three operating points listed in Table 1. Other operating conditions were investigated. The unsteady phenomenon was not present in the spectrum of any of these. Possible reasons for the presence of the phenomenon at certain operating points are discussed in the following section.

\section{Influencing parameters}

The previous discussion focused on operating points at which the described flow phenomenon occurs. It was found, however, that not all operating points actually feature this unsteady flow structure. Furthermore, no such pressure fluctuations are measured in the cavity inlet at any operating point precluding a causal link of the unsteadiness in the cavity outlet to upstream effects. Spectral analysis of accelerometer signals recorded at the rotor bearings eliminates vibrations as a source for the unsteady signals.

As depicted in Figure 9, the occurrence of unsteady flow structures in the outlet cavity appears to be dependent on the cavity flow coefficient $\varphi_{\text {cav }}$. As discussed above, the axial velocity $c_{a x}^{*}$ is not measured directly; it is a theoretical value instead. The operating points at which the unsteady flow phenomenon does occur, feature a

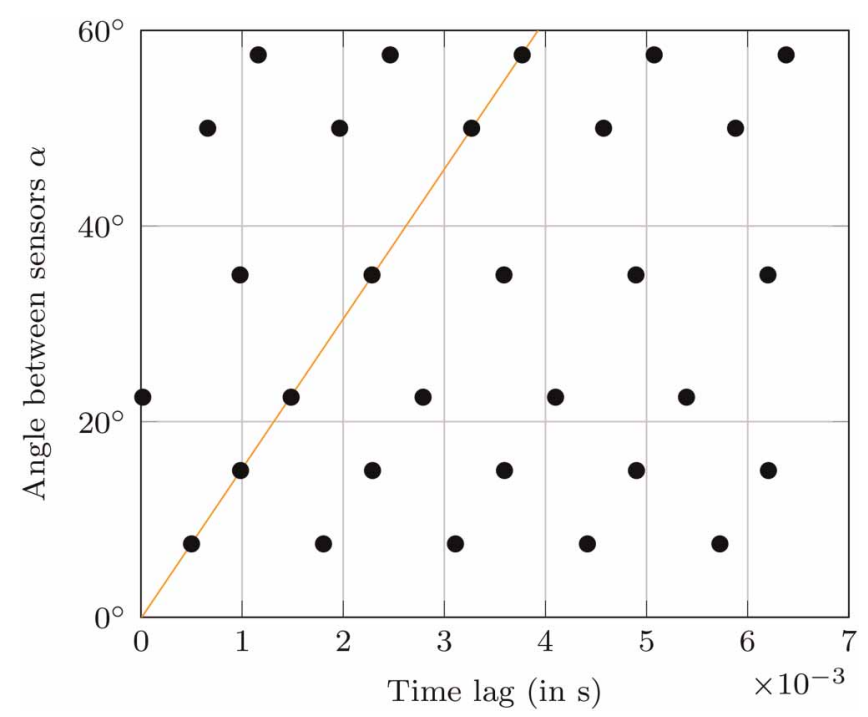

Figure 7. Cross-correlation results for the signal component in the amplitude spectrum of the ADP at EO $=6.6$. 

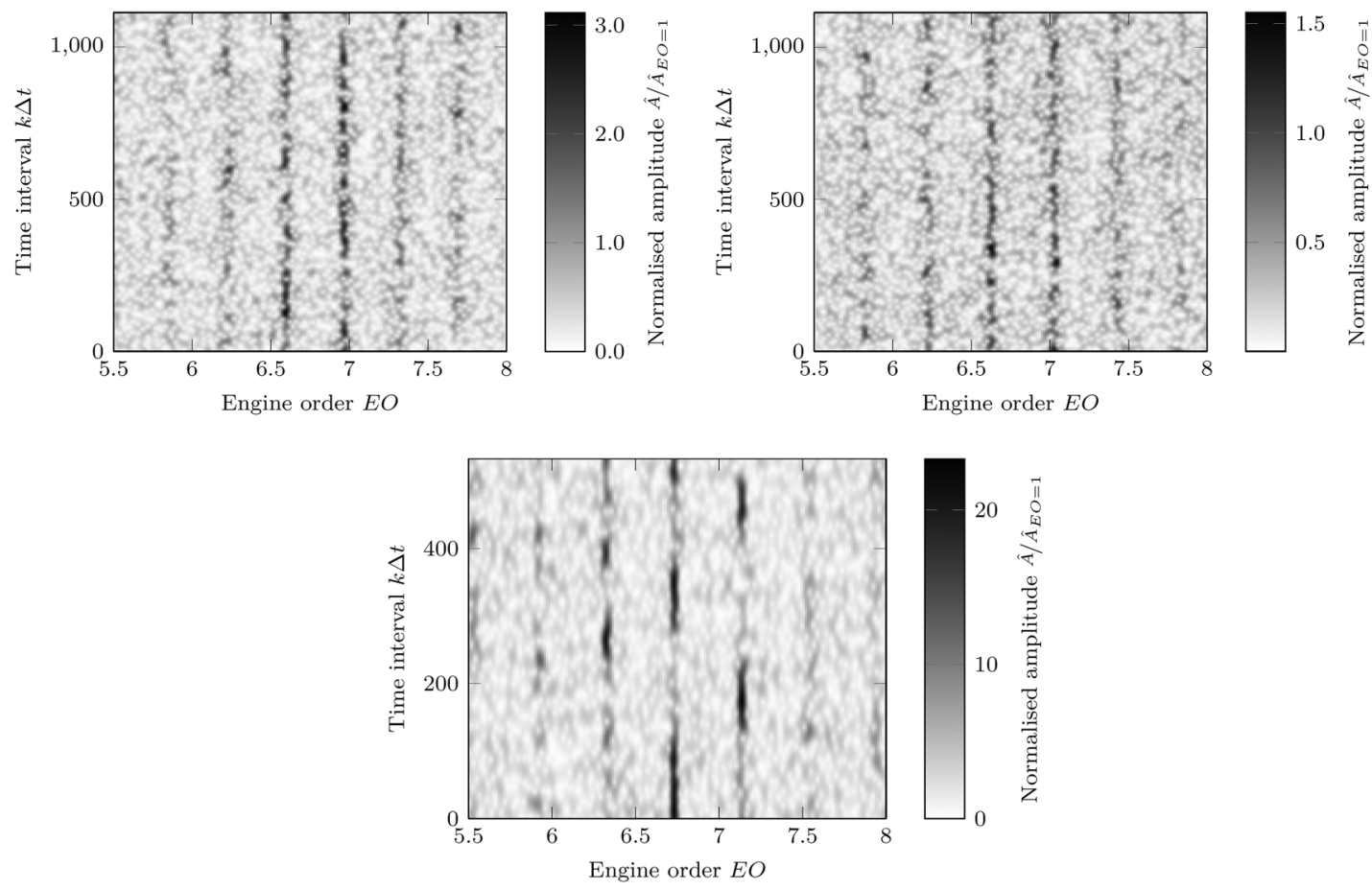

Figure 8 . Waterfall diagrams of OP 1 (ADP) with $\Delta t \approx 0.43 \mathrm{~s}$ (top left), OP 2 with $\Delta t \approx 0.43 \mathrm{~s}$ (top right) and OP 3 with $\Delta t \approx 0.86 \mathrm{~s}$ (bottom).

velocity ratio between $\varphi_{\text {cav }}=c_{a x}^{*} / U \approx 0.5 \ldots 0.75$. For operating points with $\varphi_{\text {cav }}>1$, the unsteady fluctuation is apparently suppressed. A lower boundary appears to exist as well as indicated by the operating point with $\varphi_{\text {cav }}=0.28$. The signal-to-noise ratio, as well as the relative rotational speed $\omega_{s} / \Omega$ are remarkably similar for all operating points investigated. The noise level is determined by eliminating the deterministic signal components by deconvolution (Kluge et al., 2015) of the signal. Thus isolating the deterministic components, the stochastic components of the signal remain. From those, the noise level is calculated as the root mean square of the stochastic signal components.

\section{Numerical simulation}

Similar flow phenomena are observed in URANS simulations of the test rig. To compare the experimental and numerical results, the unsteady pressure is analysed at monitor points identical in position to the unsteady

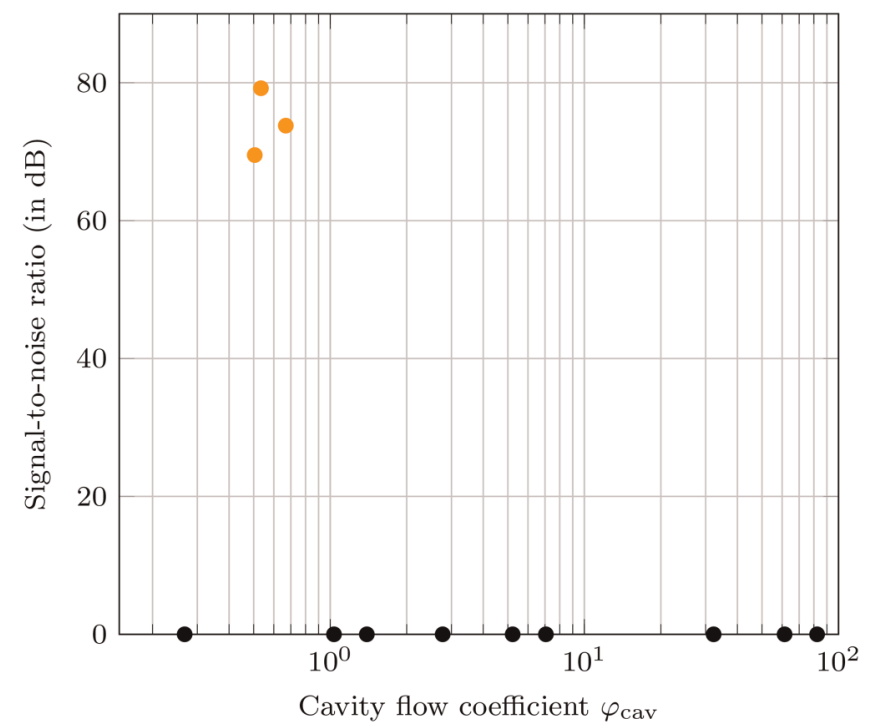

Figure 9. Signal-to-noise ratio as an indication of the presence of the unsteady flow regime. 


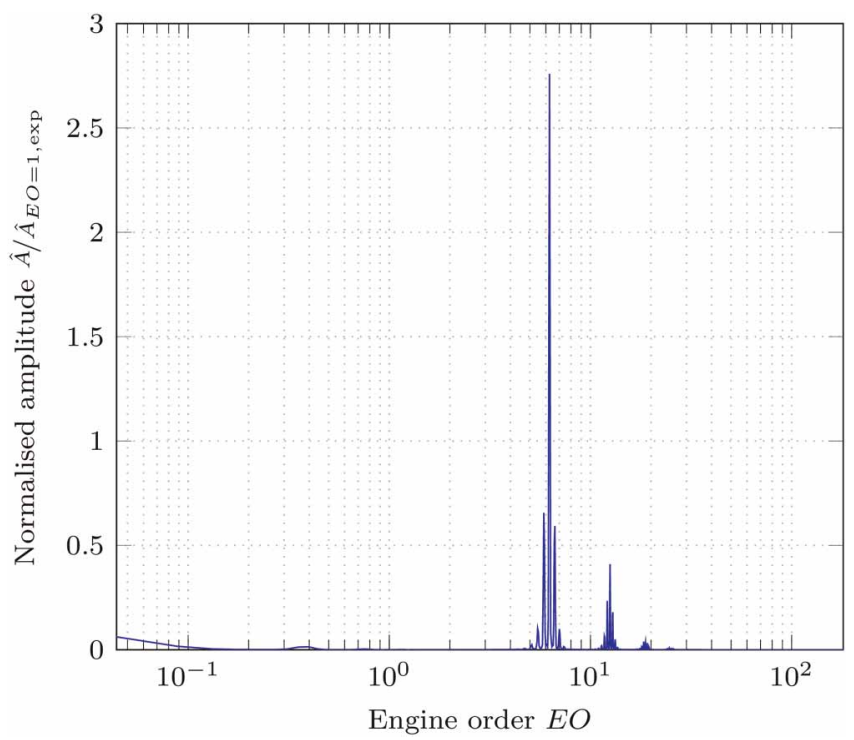

Figure 10. Amplitude spectrum of the numerical simulation at ADP (monitor point location identical to actual probe).

pressure sensors in the experiment. Figure 10 shows the amplitude spectrum of the unsteady pressure at operating point 1. There are several distinct peaks in the frequency band of $E O=5 \ldots 7.5$, forming a similar frequency pattern to the experimental data presented in Figure 6. The distance between two peaks is approximately $46.3 \mathrm{~Hz}$ compared to $42.6 \mathrm{~Hz}$ in the experimental data. Additional patterns can be seen at $E O=11 \ldots 14$, $17 \ldots 20$, and $23 \ldots 26$, though much lower in intensity. As these are multiples of the $E O$ of the non-harmonic pressure fluctuation it is likely that they are higher-order harmonics of this structure.

The amplitude is more than three times higher than that of the experiment. It is important to note that the numerical set-up describes a perfect rotor without eccentricity or other imperfections, which is why no peak at $E O=1$ exists.

The results of the cross-correlation for the peak at $E O \approx 6.6$ is displayed in Figure 11 . From the crosscorrelation, the number of nodes is derived as $N=16$ and the structure is rotating at $\omega_{s} \approx 0.39 \Omega$. The angular velocity is over-predicted by $7 \%$ compared to the experimental data while the number of nodes is in the range of the experimental results. It is important to note, however, that the accuracy of the cross-correlation is limited due to the comparatively lower sampling frequency, i.e. the time step of the simulation.

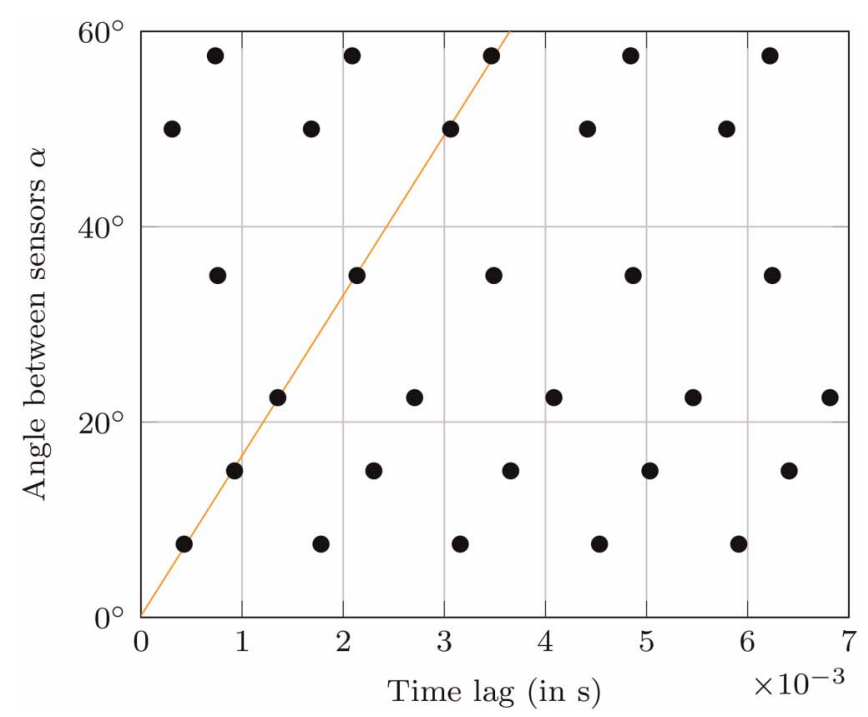

Figure 11. Cross-correlation of the pressure signal from the URANS simulation of the ADP for the signal component at $E O=6.6$ with a linear regression correlation coefficient $R^{2}=0.9997$. 


\section{Conclusions}

Unsteady pressure fluctuations are observed in the outlet of a labyrinth seal cavity modelling a tip cavity of a shrouded turbine. These fluctuations are non-harmonic to the rotor speed and a kinematic model can be used to explain the spectral signature of the phenomenon. The fluctuations are similar to observations in rim seal cavities reported in literature. Based on the data at hand, no certain conclusion with regard to the aerodynamic cause, e. g. Taylor-Couette vortices or Kelvin-Helmholtz instabilities, may be drawn. Shallow cavity modes may be eliminated as the source of the unsteadiness based on the linear frequency pattern in the amplitude spectrum. Additionally, mechanical vibration of the rotor is ruled out as the cause of the unsteady pressure.

The occurrence of the unsteady pressure fluctuations is shown to depend on the cavity flow coefficient $\varphi_{\text {cav }}$ defined in this paper, i.e. the ratio of a theoretical axial velocity of the leakage flow and the circumferential velocity of the shroud. The fluctuations are present in the signal spectrum only while this flow coefficient is in a narrow range of approximately 0.5 to 0.75 . Additional measurements and simulations are required to confirm and narrow down the limits of this range.

URANS simulations are shown to reproduce the pressure fluctuation. The spectral signature is very similar to the experimental data. There are small discrepancies in the number of nodes and the angular velocity at which the modes rotate. The discrepancies may be due to the limited sampling frequency defined by the time step of the numerical simulation. Additionally, the frequency resolution may be improved by longer simulations.

Future CFD simulations of additional operating points will serve to confirm or contradict the dependency of unsteady pressure fluctuations in the labyrinth seal cavity outlet on the ratio of axial flow velocity and rotational speed of the shroud. If confirmed, the phenomenon may be analysed further for the underlying aerodynamic causes of the fluctuation based on the simulations. A forthcoming publication will show that LES simulations are even more suitable for modelling the physical phenomena described in the present publication than RANS-based CFD.

\section{Nomenclature}

\section{Greek}

$\alpha \quad$ sensor spacing angle, deg

$\beta \quad$ nodal spacing angle, deg

$\Pi$ total pressure ratio

$\varphi_{\text {cav }}$ cavity flow coefficient $c_{a x}^{*} / U$

$\Omega$ rotor speed, $s^{-1}$

$\omega$ angular velocity, $s^{-1}$

\section{Roman}

$\hat{A} \quad$ absolute spectral amplitude

ADP aerodynamic design point

$c^{*}$ theoretical velocity, $\mathrm{m} / \mathrm{s}$

$E O$ engine order

$f$ frequency, $\mathrm{Hz}$

$N$ number of nodes

OP operating point

$R_{x y} \quad$ cross-correlation function

$t$ time, s

$U$ circumferential velocity of disc, $\mathrm{m} / \mathrm{s}$

Table 2. Overview of investigated operating points.

\begin{tabular}{|l|l|l|l|l|l|l|l|l|l|l|l|l|}
\hline Operating point & & & & & & & & & OP 3 & OP 1 & OP 2 & \\
\hline Pressure ratio $\Pi$ & 1.280 & 1.152 & 1.044 & 1.280 & 1.152 & 1.044 & 1.280 & 1.152 & 1.044 & 1.280 & 1.152 & 1.044 \\
\hline Rot. speed $\Omega / \Omega_{\mathrm{ADP}}$ & $0 \%$ & $0 \%$ & $0 \%$ & $10 \%$ & $10 \%$ & $10 \%$ & $50 \%$ & $50 \%$ & $50 \%$ & $100 \%$ & $100 \%$ & $100 \%$ \\
\hline
\end{tabular}




\section{Subscripts}

ax axial

exp experimental

res resolution

$s$ coherent structure

$S$ sampling

cav cavity

\section{Appendix}

\section{Operating points}

All operating points analysed in this study, including those, which feature no unsteady pressure fluctuations, are summarised in Table 2.

\section{Frequency resolution}

The frequency resolution in Fast Fourier Transformations (FFT)

$$
\Delta f_{\text {res }}=\frac{f_{S}}{N_{S}}
$$

is limited by the length of the signal trace, i.e. the number of samples $N_{S}$, for a given sampling frequency $f_{S}$ (cf. Bendat and Piersol, 1980).

\section{Acknowledgements}

The authors gratefully acknowledge the contribution of the DLR Institute of Propulsion Technology and MTU Aero Engines AG for providing TRACE.

\section{Funding sources}

The investigations were conducted as part of the joint research programme AG Turbo and supported by the German Federal Ministry for Economic Affairs and Energy and MTU Aero Engines AG. German Federal Ministry for Economic Affairs and Energy, 03ET7021O; MTU Aero Engines AG.

\section{Competing interests}

Tim Kluge declares that he has no conflict of interest. Iris S. Lettmann declares that she has no conflict of interest. Marcel Oettinger declares that he has no conflict of interest. Lars Wein declares that he has no conflict of interest. Joerg R. Seume declares that he has no conflict of interest.

\section{References}

Beard P. F., Gao F., Chana K. S., and Chew J. (2017). Unsteady flow phenomena in turbine rim seals. Journal of Engineering for Gas Turbines and Power. 139 (3): 032501. https://doi.org/10.1115/1.4034452.

Bendat J. S. and Piersol A. G. (1980). Engineering applications of correlation and spectral analysis. New York: Wiley.

Biester M. H.-O., Henke M., Seume J. R., Gündogdu Y., and Engel K. (2012). Unsteady wake-blade interaction: A correlation between surface pressure fluctuations and loss generation. In: Proc ASME Turbo Expo 2012, Vol. 8: Turbomachinery, Parts A, B, and C, ASME, Copenhagen, Denmark, pp. 2743-2752. GT2012-69616.

Boudet J., Hills N. J., and Chew J. W. (2006). Numerical simulation of the flow interaction between turbine main annulus and disc cavities. In 'Proc ASME Turbo Expo 2006', Vol. 6: Turbomachinery, Parts A and B, ASME, Barcelona, Spain, pp. 553-562. GT2006-90307.

Boutet-Blais G., Lefrancois J., Dumas G., J S., Harvey J.-F., et al. (2011). Passive tracer validity for cooling effectiveness through flow computation in a turbine rim seal environment. In 'Proc ASME Turbo Expo 2011', Vol. 5: Heat Transfer, Parts A and B., ASME, Vancouver, Canada, pp. 821-831. GT2011-45654.

Cao C., Chew J. W., Millington P. R., and Hogg S. I. (2004). Interaction of rim seal and annulus flows in an axial flow turbine. Journal of Engineering for Gas Turbines and Power. 126 (4): 786-793. https://doi.org/10.1115/1.1772408. 
Cumpsty N. A. (2010). Preparing for the future: Reducing gas turbine environmental impact-IGTI scholar lecture. Journal of Turbomachinery. 132 (4): 041017. https://doi.org/10.1115/1.4001221.

Darwish M. S. (1993). A new high-resolution scheme based on the normalized variable formulation. Numerical Heat Transfer, Part B: Fundamentals. 24 (3): 353-371. https://doi.org/10.1080/10407799308955898.

Gao F., Chew J. W., Beard P. F., Amirante D., and Hills N. J. (2017). Numerical studies of turbine rim sealing flows on a chute seal configuration. In 'Proc ETC 12', European Turbomachinery Society, Stockholm, Sweden. ETC2017-284.

Henke M., Biester M. H.-O., Guendogdu Y., Lippl F., Mass E., and Seume J. (2012). Numerical assisted design of a variable rotating vane carrier device for turbine test rigs with split housing structures using a segmented half-ring bearing concept. In 'Proc AIAA 2012', AIAA, Honolulu, Hawaii, pp. 4670-4679. AIAA 2012-1660.

Henke M., Wein L., Kluge T., Biester M.-O., Guendogdu Y., and Seume J. (2016). Experimental and numerical verification of the coreflow in a new low-pressure turbine. In 'Proc ASME Turbo Expo 2016', ASME, Seoul, South Korea. GT2016-57101.

Horwood J. T., Hualca F. P., Scobie J. A., Wilson M., Sangan C. M., and Lock G. D. (2018). Experimental and computational investigation of flow instabilities in turbine rim seals. Journal of Engineering for Gas Turbines and Power. 141 (1): 011028. https://doi.org/10. $1115 / 1.4041115$.

Horwood J. T., Hualca F. P., Wilson M., Scobie J. A., Sangan C. M., et al. (2020). Flow instabilities in gas turbine chute seals. Journal of Engineering for Gas Turbines and Power. 142 (2): 021019. https://doi.org/10.1115/1.4045148.

Hualca F. P., Horwood J. T., Sangan C. M., Lock G. D., and Scobie J. A. (2020). The effect of vanes and blades on ingress in gas turbines. Journal of Engineering for Gas Turbines and Power. 142 (2): 021020. https://doi.org/10.1115/1.4045149.

Jakoby R., Zierer T., Lindblad K., Larsson J., deVito L., et al. (2004). Numerical simulation of the unsteady flow field in an axial gas turbine rim seal configuration. In 'Proc ASME Turbo Expo 2004', Vol. 4: Turbo Expo 2004, ASME, Vienna, Austria, pp. 431-440. GT2004-53829.

Julien S., Lefrancois J., Dumas G., Boutet-Blais G., Caron J.-F., and Marini R. (2010). Simulations of flow ingestion and related structures in a turbine disk cavity. In 'Proc ASME Turbo Expo 2010', Vol. 4: Heat Transfer, Parts A and B, ASME, Glasgow, UK, pp. 1071-1080. GT2010-22729.

Kato M. and Launder B. E. (1993). The modeling of turbulent flow around stationary and vibrating square cylinders. In Proc 9th Symposium on Turbulent Shear Flows, Kyoto, Japan.

Kluge T., Henke M., and Seume J. (2015). Comparison of different methods for the analysis of time-resolved flow field measurements in an axial turbine. In 'Proc ETC 11', European Turbomachinery Society, Madrid, Spain. ETC2015-189.

Kluge T., Wein L., Schmierer R., and Seume J. R. (2019). Sensitivity analysis, design, instrumentation, and experimental validation of a novel labyrinth seal rig. In 'Proc ETC 13', European Turbomachinery Society, Lausanne, Switzerland. ETC2019-078.

Kügeler E. (2004). Numerisches Verfahren zur genauen Analyse der Kühleffektivität filmgekühlter Turbinenschaufeln, Forschungsbericht, Deutsches Zentrum für Luft- und Raumfahrt.

Nürnberger D. (2004). Implizite Zeitintegration für die Simulation von Turbomaschinenströmungen, Forschungsbericht, Deutsches Zentrum für Luft- und Raumfahrt.

Rabs M., Benra F.-K., Dohmen H. J., and Schneider O. (2009). Investigation of flow instabilities near the rim cavity of a 1.5 stage gas turbine. In 'Proc ASME Turbo Expo 2009', Vol. 3: Heat Transfer, Parts A and B, ASME, Orlando, FL, USA, pp. $1263-1272$. GT2009-59965.

Schaedler R., Kalfas A. I., Abhari R. S., Schmid G., and Voelker S. (2016). Modulation and radial migration of turbine hub cavity modes by the rim seal purge flow. Journal of Turbomachinery. 139 (1): 011011. https://doi.org/10.1115/1.4034416.

van Albada G. D., van Leer B., and Roberts W. W. Jr. (1982). A comparative study of computational methods in cosmic gas dynamics. Astronomy \& Astrophysics. 108: 76-84. https://doi.org/10.1007/978-3-642-60543-7_6.

Wein L., Seume J. R., and Herbst F. (2018). Unsteady flow in a labyrinth seal. In 'Proc GPPS Forum 2018', GPPS, Montreal, Canada. GPPS-NA-2018-0036.

Wein L., Kluge T., Seume J., Hain R., Fuchs T., et al. (2020). Validation of RANS turbulence models for labyrinth seal flows by means of particle image velocimetry. In 'Proc ASME Turbo Expo 2020', ASME, London, England. GT2020-14885.

Wilcox D. C. (1988). Reassessment of the scale-determining equation for advanced turbulence models. AIAA Journal 26 (11): 1299-1310. https://doi.org/10.2514/3.10041. 